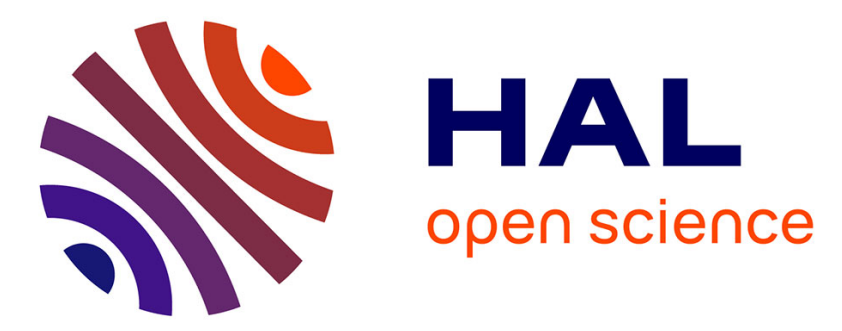

\title{
Uncovering a log-normal cascade process in high Reynolds number turbulence from wavelet analysis
}

\author{
Alain Arnéodo, Sébastien Manneville, Jean-François Muzy
}

\section{To cite this version:}

Alain Arnéodo, Sébastien Manneville, Jean-François Muzy. Uncovering a log-normal cascade process in high Reynolds number turbulence from wavelet analysis. Advances in Turbulence VII, 46, Springer, pp.215-218, 1998, Fluid Mechanics and Its Applications book series (FMIA, volume 46), 9789401151184. 10.1007/978-94-011-5118-4_53. hal-01557123

\section{HAL Id: hal-01557123 \\ https://hal.science/hal-01557123}

Submitted on 5 Jul 2017

HAL is a multi-disciplinary open access archive for the deposit and dissemination of scientific research documents, whether they are published or not. The documents may come from teaching and research institutions in France or abroad, or from public or private research centers.
L'archive ouverte pluridisciplinaire HAL, est destinée au dépôt et à la diffusion de documents scientifiques de niveau recherche, publiés ou non, émanant des établissements d'enseignement et de recherche français ou étrangers, des laboratoires publics ou privés. 


\section{UNCOVERING A LOG-NORMAL CASCADE PROCESS IN HIGH REYNOLDS NUMBER TURBULENCE FROM WAVELET ANALYSIS}

A. ARNEODO, S. MANNEVILLE AND J.F. MUZY

Centre de Recherche Paul Pascal, Univ. Bordeaux I

avenue Schweitzer, 33600 Pessac, France

We present a generalization of the Castaing et al. [1] approach of velocity intermittency using the wavelet transform (WT). This description consists in looking for a multiplicative cascade process directly on the velocity field assuming that the probability density function (pdf) of the modulus maxima of the WT (WTMM) at a given scale $a, P_{a}(T)$, can be expressed as a weighted sum of dilated pdf's at a larger scale $a^{\prime}>a$ :

$$
P_{a}(T)=\int G_{a a^{\prime}}(u) P_{a^{\prime}}\left(\mathrm{e}^{-u} T\right) \mathrm{e}^{-u} \mathrm{~d} u, \text { for } \boldsymbol{a}^{\prime}>\boldsymbol{a} .
$$

The reader is referred to Ref. [2] for the computation of the WT of the velocity field and the restriction of the analysis to the modulus maxima of the WT (WTMM method). Our numerical method for estimating the Fourier transform $\widehat{G}_{a a^{\prime}}$ of the kernel $G_{a a^{\prime}}$ is described in Refs. [3, 4]. This wavelet-based method is applied to a turbulent velocity signal recorded in the Modane wind tunnel and kindly provided by Y. Gagne and Y. Malecot. The Taylor scale based Reynolds number is $R_{\lambda} \simeq 2000$ and the sample is $2.5 \cdot 10^{8}$ points long, with a resolution of roughly $3 \eta$ (where $\eta$ is the Kolmogorov scale), corresponding to 25000 integral scales. We then propose a two-point statistical analysis based on space-scale correlations [5].

\section{Experimental results of the one-point statistical approach}

The computation of $\widehat{G}_{a a^{\prime}}$ for various pairs of inertial scales $a<a^{\prime}$ reveals the existence of a function $s\left(a, a^{\prime}\right)$ and of a single kernel $\widehat{G}$ such that $\widehat{G}_{a a^{\prime}}=$ $\widehat{G}^{s\left(a, a^{\prime}\right)}$ (i.e. formally $\left.G_{a a^{\prime}}=G^{\otimes s\left(a, a^{\prime}\right)}\right)$. According to the definitions given in Ref. [1], this means that the underlying cascade process is self-similar 

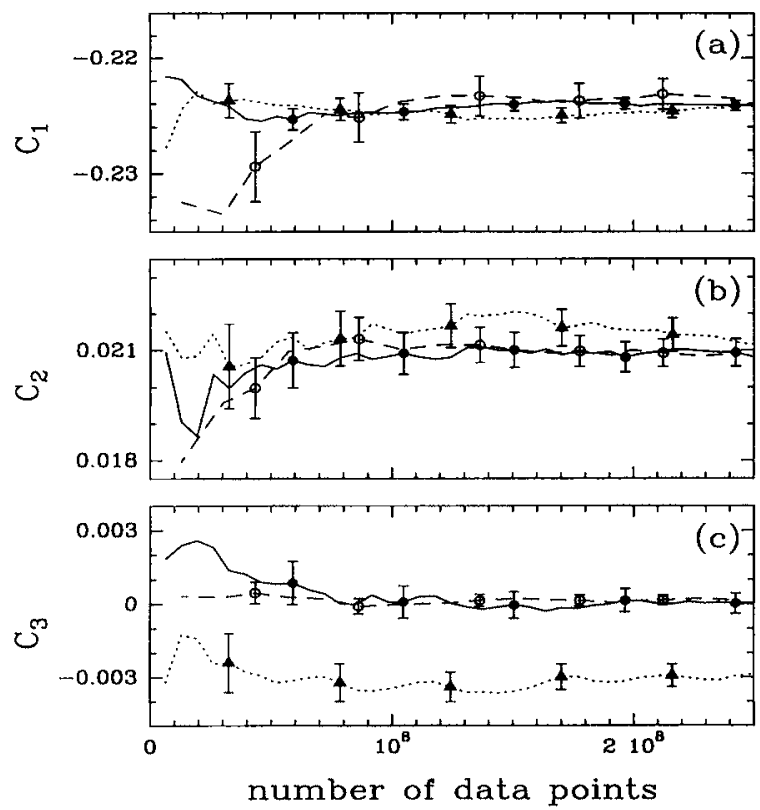

Figure 1. The first three cumulants of $G_{a a^{\prime}}$ vs the sample length. Turbulent velocity signal for $a=770 \eta$ and $a^{\prime}=1540 \eta$ ( $\circ$ and dashed line), log-normal numerical process with $m=0.32$ and $\sigma^{2}=0.03$ ( $\bullet$ and solid line) and log-Poisson numerical process with $\lambda=2, \delta=0.89$ and $\gamma=-0.082$ (triangles and dots) for the two corresponding scales $a=2^{8}$ and $a^{\prime}=2^{9}$. The analyzing wavelet is a first order compactly-supported wavelet [4].

and that $s\left(a, a^{\prime}\right)$ accounts for the number of cascade steps from scale $a^{\prime}$ to scale $a$. Such a cascade is said scale-similar (or scale-invariant) if $s\left(a, a^{\prime}\right)=$ $\ln \left(a^{\prime} / a\right)$. In the present case, $s\left(a, a^{\prime}\right)$ turns out to be very well fitted by the functional form $s\left(a, a^{\prime}\right)=\left(a^{-\beta}-a^{\prime-\beta}\right) / \beta$, where $\beta \simeq 0.095$ quantifies the departure from scale-similarity (scale-invariance is restored for $\beta \rightarrow 0$ ) [6]. Thus, the cascade process is self-similar but not scale-invariant.

To analyze precisely the shape of $G$, we use the Taylor series expansion: $\widehat{G}(p)=\exp \left(\sum_{k=1}^{\infty} c_{k} \frac{(\mathrm{i} p)^{k}}{k !}\right)$, where the (real valued) coefficients $c_{k}$ are the cumulants of $G$. Figure 1 shows the first three cumulants $C_{k}=s\left(a, a^{\prime}\right) c_{k}$ (for a given pair of inertial scales $a<a^{\prime}$ ) for the turbulence data and for both a log-normal (mean $m$ and variance $\sigma^{2}$ ) and a log-Poisson (with the $\lambda, \delta$ and $\gamma$ parameters consistent with the ones proposed by She and Leveque [7]) synthetic numerical processes. Even though both numerical processes perfectly fit the first two cumulants, the log-Poisson model yields a third order cumulant that is more than one order of magnitude higher than the experimental one, whereas the very small (theoretically zero) third order cumulant of the log-normal numerical process still remains within the 

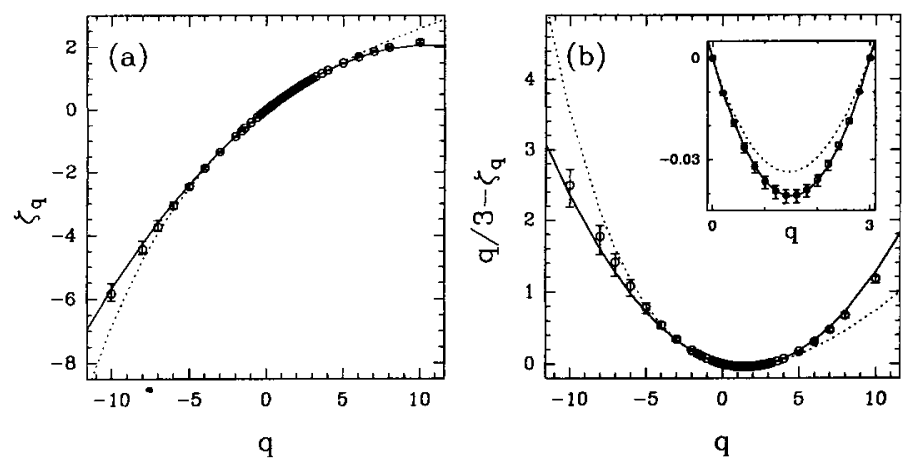

Figure 2. WTMM estimation of the $\zeta_{q}$ spectrum for the turbulent velocity signal [4]. (a) $\zeta_{q}$ vs $q$. (b) Deviation of the experimental spectrum from $q / 3$. The experimental measurements (o) are compared to the theoretical quadratic ESS spectrum of a $\log$-normal process with $m=0.32$ and $\sigma^{2}=0.03$ (solid line) and to the She and Leveque [7] log-Poisson prediction (dots).

error bars of the experimental one. We thus exclude log-Poisson statistics as proposed in Ref. [7] and conclude that the statistics underlying the velocity fluctuations are log-normal, as soon as $R_{\lambda}>1000$.

Testing the convolution formula (1) on the WTMM pdf's using a Gaussian kernel yields results in very good agreement with the log-normal cascade picture [4]. A second test of log-normality lies in the scaling exponents $\zeta_{q}$ of the velocity structure functions. As shown in Fig. 2(a), the experimental spectrum, obtained using extended self-similarity (ESS) [8] and extended to negative values of $q$ thanks to the WTMM method, remarkably coincides with the quadratic log-normal prediction. The log-Poisson prediction [7] provides a good approximation of $\zeta_{q}$ for $q \in[-6,6]$. However, plotting the deviation of the $\zeta_{q}$ 's from the Kolmogorov (1941) linear $q / 3$ spectrum (Fig. 2(b)), reveals a systematic departure of the log-Poisson prediction from the experimental spectrum, whereas the log-normal model still perfectly fits the experimental data.

\section{Space-scale correlations of the WT: a two-point statistical analysis}

To get a deeper insight into the nature of the statistics, we study the spacescale correlations of the "magnitude" $\omega(x, a)$ of the velocity field at point $x$ and scale $a$ [5]. $\omega(x, a)$ is defined as the logarithm of a local average over a size $a$ of the wavelet coefficients at scale $a$ around the point $x$. Here, we use the velocity increments to compute $\omega(x, a)$. Figure 3 shows various correlation functions $C\left(\Delta x, a, a^{\prime}\right)=\overline{\tilde{\omega}(x, a) \tilde{\omega}\left(x+\Delta x, a^{\prime}\right)}$, where the overline stands for ensemble average and $\tilde{\omega}$ for the centered process 

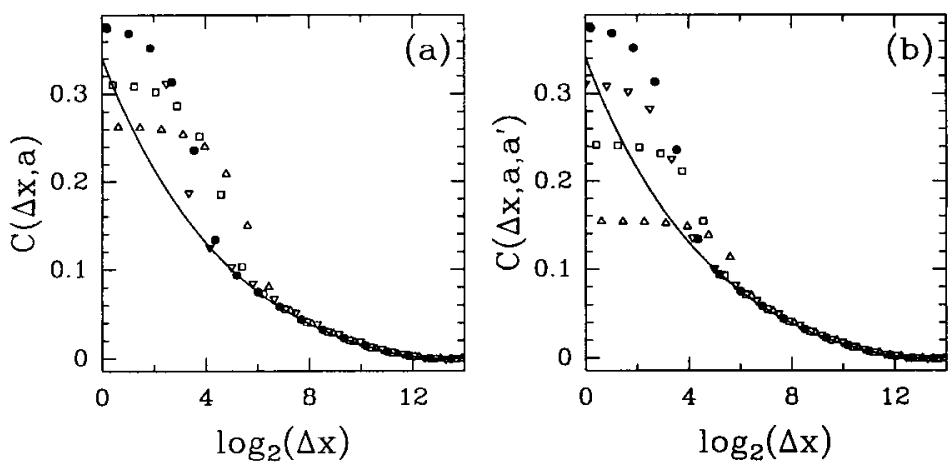

Figure 3. Increment magnitude correlation functions of the turbulent velocity signal. (a) "One-scale" correlation functions $C(\Delta x, a)=C(\Delta x, a, a)$ at scale $a=8$ (down triangles), $16(\bullet), 32$ (squares) and 64 (up triangles). (b) "Two-scale" correlations functions at scale $a=8, a^{\prime}=16$ (down triangles), $a=16, a^{\prime}=16(\bullet)$, $a=16, a^{\prime}=32$ (squares) and $a=16, a^{\prime}=64$ (up triangles). The solid line corresponds to a non scale-invariant, $\log$-normal cascade model with $\beta=0.3, \sigma^{2}=0.27$. $a, a^{\prime}$, and $\Delta x$ are expressed in mesh size $(\simeq 3 \eta)$ units.

$\omega-\bar{\omega}$. Once again, the experimental results are in very good agreement with a log-normal, non scale-invariant cascade. However, the cross-over from the value $C(\Delta x=0, a, a)$ down to the fitted curve and the fact that the scaleinvariance breaking exponent $\beta$ takes a different value when computed from the space-scale correlations of the increment magnitude $(\beta=0.3)$ and from the WTMM estimation of the kernel $G(\beta=0.095)$ suggest that simple (even non scale-invariant) self-similar cascades are not sufficient to account for the space-scale structure of the velocity field.

\section{References}

1. Castaing B., Gagne Y. and Hopfinger E. J., Physica D 46 (1990) 177; Chabaud B., Naert A., Peinke J., Chillà F., Castaing B. and Hebral B., Phys. Rev. Lett. 73 (1994) 3227; Castaing B. and Dubrulle B., J. Phys. II France 5 (1995) 895.

2. Muzy J.F., Bacry E. and Arneodo A., Phys. Rev. Lett. 67 (1991) 3515; Int. J. Bifurcation and Chaos 4 (1994) 245; Arneodo A., Bacry E. and Muzy J.F., Physica A 213 (1995) 232.

3. Arneodo A., Muzy J.F. and Roux S.G., J. Phys. II France 7 (1997) 363.

4. Arneodo A., Manneville S. and Muzy J.F., Eur. Phys. J. B 1 (1998) 129.

5. A. Arneodo, E. Bacry, S. Manneville and J.F. Muzy, Phys. Rev. Lett. 80 (1998) 708.

6. The reader may find more quantitative results on the breaking of scale-invariance in different flow configurations in Refs. [4]. In particular, the exponent $\beta$ is found to decrease with $R_{\lambda}$, strongly indicating that the non scale-similar multiplicative process asymptotically converges towards a scale-similar cascade.

7. She Z.S. and Leveque E., Phys. Rev. Lett. 72 (1994) 336.

8. Benzi R., Ciliberto S., Trippiccione R., Baudet C., Massaioli F. and Succi S., Phys. Rev. E 48 (1993) R29; Benzi R., Ciliberto S., Baudet C., Ruiz-Chavarria G. and Tripiccione R., Europhys. Lett. 24 (1993) 275. 